Servir e proteger:

\section{determinantes da avaliação pública \\ sobre a qualidade do trabalho \\ das Polícias Militares no Brasil}

Recebido: 11.02.16

Aprovado: 10.01 .17
Luís Felipe Zilli

\& Vinícius Assis Couto*

Resumo: Inserido no campo de estudo das relações entre sociedade civil e instituições de segurança pública, o presente artigo tem como objetivo mensurar e analisar a influência que determinados fatores - como, por exemplo, características sociodemográficas, experiências de vitimização criminal e/ou institucional, ou mesmo o contato direto com agentes policiais - exercem sobre a forma como a população brasileira avalia a qualidade do trabalho das Polícias Militares no país. Para tanto, foram utilizados dados produzidos pela Pesquisa Nacional de Vitimização (PNV), estudo por amostragem realizado entre 2010 e 2012 em todos os estados brasileiros. De modo geral, o que a análise indica é que, ao contrário do que preconiza o senso comum, o fato de ter sido ou não vítima de crimes, tradicional indicador de eficácia do trabalho policial, exerce relativamente pouca influência sobre a opinião que a população tem sobre a qualidade do trabalho das Polícias Militares. As variáveis que apresentaram os maiores efeitos sobre a avaliação (positiva ou negativa) das PMs foram aquelas relacionadas ao fato de ter sido vítima de violência ou extorsão por parte dos próprios militares, bem como as experiências de contato direto com os policiais. Tais achados reforçam a hipótese, bastante difundida na literatura internacional, de que avaliações positivas ou negativas sobre a qualidade do trabalho das forças policiais não se encontram exclusiva e necessariamente vinculadas à percepção de que tais corporações são mais ou menos eficazes em coibir a ocorrência de crimes, mas sim a questões localizadas unicamente na esfera institucional destas organizações, como, por exemplo, o combate à violência e à corrupção praticadas pelos próprios PMs e à melhoria do atendimento prestado a segmentos populacionais mais vulneráveis.

Palavras-chave: segurança pública, organizações policiais, avaliação e accountability, Polícia Militar, políticas públicas.

\title{
Introdução
}

$$
\text { A }
$$
o longo dos últimos anos, o debate público sobre o papel do Estado no Brasil tem enfatizado duas ordens de questões: por um lado, o aumento da pressão popular pelo incremento dos níveis de eficiência e eficácia das políticas públicas; por outro, o fato de que tais exigências encontram-se cada vez mais atreladas à percepção de que esses ganhos precisam se fazer acompanhar de maiores níveis de transparência e accountability, garantidos pelo funcionamento de mecanismos

\author{
* Luís Felipe Zilli é \\ doutor em sociologia \\ pela Universidade \\ Federal de Minas \\ Gerais (UFMG) e \\ pesquisador em \\ ciência e tecnologia \\ da Fundação João \\ Pinheiro (FJP/MG). \\ Dentro da instituição, \\ é vinculado à \\ Diretoria de Políticas \\ Públicas (DPP) e ao \\ Núcleo de Estudos \\ em Segurança \\ Pública (Nesp). \\ <felipe.zilli@fjp. \\ mg.gov.br>. \\ Vinícius Assis Couto é \\ doutor em sociologia \\ pela Universidade \\ Federal de Minas \\ Gerais (UFMG) e \\ pesquisador do \\ Centro de Estudos \\ de Criminalidade e \\ Segurança Pública \\ (Crisp/UFMG). \\ <viniccouto@gmail. \\ com>.
}


1. Pesquisa recente do Fórum Brasileiro de Segurança Pública (FBSP) indicou que grande parte dos policiais brasileiros é favorável à reforma estrutural de suas instituições e do Sistema de Justiça Criminal. Entre as questões mais urgentes levantadas pelo estudo encontram-se temas como a integração ou unificação das polícias Civil e Militar (polícia de ciclo completo), reformulação dos planos de carreira, desmilitarização das PMs, controle externo da atividade policial, entre outros. institucionais de governança. Mais do que uma atuação eficiente e eficaz, exige-se do poder público maior transparência e mais espaço para a participação popular nos processos decisórios (Belli, 2004; Souza, 2006; Gonçalves, 2005; Moisés, 2005; Costa \& Lima, 2014).

Na área da segurança pública, por exemplo, tais demandas têm se materializado em aumento da pressão social sobre as organizações policiais e seus modos de trabalho. Especialmente na América Latina, as polícias têm sido cada vez mais cobradas não apenas a oferecer respostas mais eficientes e eficazes para o controle da violência e da criminalidade, mas também - e talvez principalmente - a executar tais tarefas de modo mais transparente, com maiores graus de legitimidade, de permeabilidade às demandas sociais e com ações sempre balizadas no respeito aos direitos civis (Tyler, 2001; Cano, 2006; Velásquez, 2006; Lopes, 2010).

É dentro deste contexto mais amplo que, ao longo das últimas décadas, diversos governos ao redor do mundo têm conduzido profundos processos de reestruturação de suas organizações policiais. Reformas como a das polícias de Nova York, Los Angeles, Boston e Bogotá, por exemplo, notabilizaram-se pelo uso intensivo de tecnologias da informação para instrumentalizar processos de gestão de atividades operacionais. Além disso, tais investimentos se fizeram acompanhar por políticas estruturadas de aproximação das organizações policiais com suas comunidades locais, visando a ampliação de seus níveis de governança e accountability (Veloso \& Ferreira, 2007; Weisburd \& Braga, 2009).

No Brasil, o debate sobre a necessidade de se submeter as forças policiais a reformas estruturais tem se intensificado ao longo dos últimos anos e, de certo modo, já apresenta uma agenda consensual entre policymakers e operadores do Sistema de Justiça Criminal ${ }^{1}$. Para além das questões eminentemente estruturais, focadas principalmente na rediscussão do modelo dual de polícia (estabelecido pelo artigo 144 da Constituição Federal de 1988, que divide a atividade policial no Brasil entre a Polícia Militar e a Polícia Civil), colocam-se também problemas de ordem incremental, como, por exemplo, o uso sistemático e intensivo de tecnologias da informação e a adoção de modelos gerenciais mais eficientes e transparentes. De certo modo, pode-se dizer que, atualmente, a discussão volta-se mais para as bases legais, políticas e institucionais adequadas à condução destes processos, do que propriamente para a sua necessidade ou pertinência. 


\section{Avaliação de desempenho e confiança nas polícias}

Todas as questões pontuadas até aqui têm como fundamento o pressuposto de que, em sociedades nas quais vigem regimes democráticos consolidados, existe forte controle social dos serviços e das instituições públicas. O próprio processo de planejamento de políticas públicas (e a consequente atuação dos órgãos públicos encarregados de implementá-las) depende, em grande medida, da legitimidade que tais ações e organizações desfrutam junto à população. Daí a importância de se compreender melhor, por exemplo, as mais diversas questões relacionadas às percepções e avaliações que a população faz dos serviços de segurança pública, bem como os níveis de confiança e legitimidade que atribui às instituições encarregadas de executá-los.

Em diversos países da América Latina, especialmente naqueles que experimentaram a vigência relativamente recente de regimes autoritários, a desconfiança popular com relação às organizações policiais é bastante recorrente. Levantamento realizado entre 2005 e 2009 pelo World Values Survey (WVS, 2011) demonstrou que, entre 56 países pesquisados, o Brasil é o quadragésimo em níveis de confiança na polícia, com aproximadamente 55\% de sua população afirmando

FIGURA 1 CONFIANÇA NA POLÍCIA

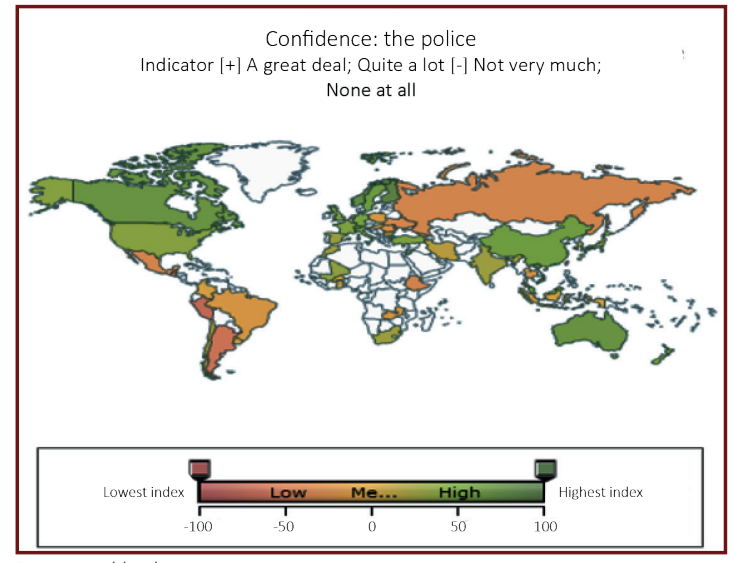

Fonte: World Values Survey, 2011 que "não confia nada" ou "confia pouco" nas organizações de força. O mapa da Figura 1 ilustra os resultados obtidos pelo levantamento realizado pelo WVS, no que diz respeito aos níveis de confiança que as populações depositam em suas organizações policiais.

É por isso que, em diversos países, pesquisas que tratam das percepções públicas sobre as organizações e o trabalho policial são utilizadas como instrumentos de aprimoramento gerencial e de accountability destas instituições, fomentando o desenvolvimento de intervenções e programas de atuação com maior legitimidade e suporte público (Soares, 2000; Veloso \& Ferreira, 2007; Sherman et alii 1998). 


\begin{abstract}
A realização e a análise de pesquisas de opinião sobre atuação policial são relevantes na medida em que podem ser utilizadas como proxy do desempenho de policiais e seus serviços e da qualidade de sua interação com as comunidades, além de auxiliarem os gestores na ordenação de prioridades, a partir das avaliações dos programas de policiamento. Cidadãos insatisfeitos com a polícia são menos tendenciosos a contatá-la ou fornecer informações sobre atividades criminosas. Percepções negativas da polícia contribuem para a redução da eficiência da própria atuação policial, o que eventualmente pode colaborar para o aumento das taxas de crimes (Oliveira Júnior, 2011: 7).
\end{abstract}

Ainda que de modo bastante esquemático, é possível dividir os estudos que tratam da avaliação pública das forças policiais em dois grandes campos: de um lado, aqueles que tratam das percepções e avaliações sobre o desempenho e a qualidade da atuação dos órgãos de segurança; de outro, pesquisas que discutem as diversas dimensões associadas à confiança que a população deposita nestas instituições (Bayley \& Skolnick, 2001; Brown \& Benedict, 2002; Goldsmith, 2005; Frank et alii, 2005; Larsen \& Blair, 2009). Além disso, muitos são, obviamente, os estudos que transitam entre as duas vertentes, avaliando em que medida avaliações de desempenho e qualidade da atuação das polícias relacionam-se com diferentes níveis de confiança nas organizações (Lopes, 2010; Oliveira Júnior, 2011; Silva \& Beato, 2013).

Em uma vasta revisão bibliográfica sobre a temática, Brown e Benedict (2002), por exemplo, identificaram que, tanto nos Estados Unidos como em diversos países europeus, quatro tipos de variáveis encontram-se mais fortemente correlacionadas a maiores ou menores níveis de confiança nas organizações policiais e, paralelamente, na melhor ou pior avaliação da qualidade de seu trabalho:

1. faixa etária,

2. contato com a polícia,

3. tipo de vizinhança,

4. cor/raça.

A partir da análise de mais de 100 estudos publicados desde os anos 1930, os autores apontam que, de modo geral, a literatura científica indica que pessoas mais "jovens", "não brancas", moradoras de vizinhanças com "menor status socioeconômico" e com contatos mais frequentes com as polícias tendem a ter menores níveis de confiança nas organizações policiais e a avaliar pior a qualidade de seu trabalho (Brown \& Benedict, 2002). 
Outros estudos problematizam o estabelecimento de relação direta entre variáveis sociodemográficas (idade, raça/cor, status socioeconômico etc.) e as dimensões da confiança e da avaliação da qualidade do trabalho policial, para atribuir maior peso às variáveis de contato direto entre a população e as organizações. Segundo essa linha de pesquisa, o principal determinante das percepções públicas sobre o trabalho policial seria o modo como as corporações atuam e se relacionam com seus mais variados públicos.

Nesse sentido, pessoas "jovens", "não brancas" e de menor "status socioeconômico" tenderiam a confiar menos nas polícias e a avaliar pior o seu trabalho devido não apenas à maior frequência com que a polícia atuaria especificamente junto a esses públicos, mas também às formas como se dão tais intervenções. O argumento central, portanto, é o de que grande parte do poder explicativo que variáveis como "idade", "raça/cor" e "status socioeconômico" exercem sobre os níveis de "confiança na polícia" viria, na verdade, do efeito interveniente da variável "contato com a polícia" (Frank et alii, 2005; Larsen \& Blair, 2009; Avdija, 2010).

Uma terceira linha de pesquisas avalia o impacto que diferentes níveis de transparência e governança na gestão das organizações policiais projetam sobre a confiança e a avaliação que a população faz sobre a qualidade da atuação das corporações. Estudo realizado por Ivkovic (2008), por exemplo, identificou duas ordens de fatores que possuem forte poder de determinação sobre estas dimensões: o contato pessoal das pessoas com as organizações policiais e os níveis de governança e transparência na gestão dessas instituições. De acordo com o estudo, que utiliza dados de um survey realizado em 28 países (Europa e América do Norte), pessoas com maior frequência de contatos com a polícia tendem a confiar menos nestas instituições. Por outro lado, em países em que as organizações policiais investiram no aumento de seus níveis de transparência e de participação popular nos processos decisórios, a população tende a avaliar melhor a qualidade do trabalho e a eficácia das instituições no enfrentamento dos problemas de segurança pública (Ivkovic, 2008).

Apesar de ser um campo de estudos bastante consolidado no exterior, ainda não é possível dizer que exista no Brasil uma tradição de pesquisas a respeito das percepções e avaliações sociais sobre os órgãos de segurança pública em geral ou, mais especificamente, sobre as organizações policiais. No entanto, a temática tem recebido cada vez mais atenção por parte de instituições acadêmicas e agências governamentais, com um recente leque de estudos que, aos moldes do que ocorre no contexto internacional, busca compreender melhor quais variáveis (demográficas, socioeconômicas, de contato etc.) estariam prioritariamente associadas a diferentes padrões perceptivos e atitudinais com relação às organizações policiais. 
2. Realizadas por meio de metodologia de survey (que consiste, basicamente, na aplicação de questionários estruturados em uma amostra representativa de determinada população), as pesquisas de vitimização têm como objetivo obter dados sobre o número e as características dos crimes sofridos pelos entrevistados. Trata-se de um instrumento bastante abrangente de aferição da vitimização criminal, permitindo mesmo mensurar a quantidade aproximada de crimes que não chegam ao conhecimento da polícia (as chamadas "cifras obscuras"). Diferentemente dos registros oficiais, organizados prioritariamente para fins de gerenciamento do trabalho das instituições do sistema de justiça criminal, os surveys de vitimização são um instrumento de pesquisa elaborado com metodologia especificamente voltada para a mensuração das experiências de vitimização criminal da população pesquisada. Isso faz com que consigam revelar, com considerável confiabilidade, as taxas de crimes que não se convertem em registros policiais,
Pesquisa realizada por Lopes (2010), por exemplo, utiliza dados de um survey de abrangência nacional para corroborar a hipótese, já discutida por estudos internacionais, de que a dimensão do contato direto com as polícias seria a principal preditora dos níveis de confiança nas instituições, juntamente com a percepção a respeito da qualidade e eficácia de seu trabalho no enfrentamento à criminalidade. Segundo os dados da pesquisa, quanto maior o contato com agentes policiais e pior a percepção de sua eficácia na prevenção dos crimes, menor será a confiança nas polícias (Lopes, 2010).

Ao utilizar também dados de um survey de abrangência nacional, Oliveira Júnior (2011) observa que, no Brasil, a dimensão da confiança nas instituições policiais encontra-se muito mais correlacionada à percepção de seus níveis de eficiência e eficácia do que propriamente a características sociodemográficas da população. Segundo o autor, a percepção de que a polícia consegue executar bem suas funções de enfrentamento da criminalidade e prevenção da violência possuiria maior impacto positivo nos níveis de confiança atribuídos à polícia do que qualquer característica individual (como idade, raça/cor, status socioeconômico, etc.).

Resultados bastante parecidos foram obtidos por pesquisa desenvolvida por Silva e Beato (2013), ainda a partir de dados de survey realizado na região metropolitana de Belo Horizonte. De acordo com os autores, a variável de maior efeito sobre a dimensão da confiança nas polícias seria a percepção de que elas são eficientes em solucionar problemas relacionados à violência. O estudo também constata que a variável "contato com a polícia" impacta significativamente os níveis de confiança nas instituições, sobretudo se este contato for iniciado pelos próprios agentes (Silva \& Beato, 2013).

O que se observa, portanto, é que, aos moldes do que já ocorre no contexto internacional, começa a se consolidar no Brasil uma linha de estudos bastante consistente sobre as percepções e avaliações que a sociedade faz a respeito das organizações policiais, sua eficiência, eficácia e modos de atuação. Conforme observado anteriormente, a compreensão mais acurada desses processos contribui para a melhoria das organizações e do trabalho policial no país, bem como para o aperfeiçoamento das políticas de segurança pública.

Com o objetivo de contribuir para esta discussão, o presente artigo utilizará dados da Pesquisa Nacional de Vitimização (PNV)² para tentar mensurar a influência que uma série de variáveis (características sociodemográficas, experiências de vitimização criminal e/ou institucional, contato com as instituições etc.) exerce sobre a conformação de padrões de avaliação da qualidade do trabalho das Polícias Militares no 
Brasil. Conforme discutido anteriormente, compreender melhor quais elementos influenciam a avaliação que a população faz sobre a qualidade do trabalho policial é condição importante para explicar os níveis de confiança que a sociedade deposita nestas corporações e, em última instância, a maior ou menor legitimidade das instituições de segurança pública.

\section{Dados e metodologia}

Financiada pelo Ministério da Justiça (MJ) entre os anos de 2010 e 2012, a Pesquisa Nacional de Vitimização foi realizada junto a uma amostra representativa da população brasileira, com idade igual ou superior a 16 anos, e moradora de cidades com mais de 15 mil habitantes em sua área urbana. O levantamento, conduzido pelo Centro de Estudos de Criminalidade e Segurança Pública da Universidade Federal de Minas Gerais (Crisp/UFMG), em parceria com o Instituto de Pesquisas Datafolha, foi feito em 346 municípios de todos os estados da federação, formando uma base de dados composta por 78.008 questionários estruturados, divididos em módulos temáticos.

De modo geral, tratou-se de um primeiro esforço de âmbito nacional dedicado não apenas a mensurar os reais índices de vitimização criminal da população brasileira, mas também as percepções públicas sobre questões como sensação de segurança, confiança nas organizações policiais e avaliação da qualidade da atuação das instituições do Sistema de Justiça Criminal.

Tendo como base os dados produzidos pela PNV, buscou-se aqui construir um modelo estatístico que mensurasse os efeitos que quatro dimensões exercem sobre a avaliação que a população brasileira faz da qualidade da atuação das Polícias Militares no país. São eles:

1. características sociodemográficas da população;

2. vitimização criminal e/ou institucional;

3. contato direto com a polícia; e

4. percepção da atuação ou contato indireto com a polícia.

Para a construção e mensuração de cada uma destas dimensões, aplicou-se a técnica da análise fatorial, procedimento que permitiu transformar as muitas variáveis que tratavam das questões de interesse em basicamente oito constructos, conforme representado na tabela a seguir: a distribuição espacial dos delitos, sua frequência e incidência, bem como uma grande diversidade de aspectos relacionados à dimensão contextual da criminalidade (perfi sociodemográficos das vítimas, rotinas e práticas correlacionadas a maiores ou menores taxas de vitimização, experiências e percepções de contato das vítimas com instituições do Sistema de Justiça Criminal etc.). 
TABELA 1

RELAÇÃO DE DIMENSÕES E FATORES CUJOS EFEITOS SOBRE A AVALIAÇÃO DA QUALIDADE DE ATUAÇÃO DAS PMS SE PRETENDE MENSURAR

\begin{tabular}{|c|c|c|}
\hline Dimensão & Variáveis integrantes & Fator gerado \\
\hline $\begin{array}{c}\text { Características } \\
\text { Sociodemográficas } \\
\text { da População }\end{array}$ & $\begin{array}{c}\text { Raça/cor } \\
\text { Escolaridade } \\
\text { Renda }\end{array}$ & $\begin{array}{c}\text { DEMO } \\
\text { (Características } \\
\text { Sociodemo- } \\
\text { gráficas) }\end{array}$ \\
\hline \multirow{3}{*}{$\begin{array}{l}\text { Vitimização Criminal } \\
\text { e/ou Institucional }\end{array}$} & Vítima de violência física por parte da PM (p.175a) - Sim / Não & \multirow{3}{*}{$\begin{array}{l}\text { VITPM } \\
\text { (Vitimização por } \\
\text { parte da PM) }\end{array}$} \\
\hline & $\begin{array}{l}\text { Vítima de insulto ou agressão verbal por parte da PM (p.175b) - } \\
\text { Sim / Não }\end{array}$ & \\
\hline & Vítima de extorsão/propina por parte da PM (p.175c) - Sim / Não & \\
\hline \multirow{7}{*}{$\begin{array}{l}\text { Vitimização Criminal } \\
\text { e/ou Institucional }\end{array}$} & Vítima de ofensa verbal (p.30a) - Sim / Não & \multirow{5}{*}{$\begin{array}{l}\text { VITPE } \\
\text { (Vitimização crimes } \\
\text { contra a pessoa) }\end{array}$} \\
\hline & Vítima de batida, empurrão ou chute (p.30e) - Sim / Não & \\
\hline & $\begin{array}{l}\text { Vítima de lesão provocada por algum objeto que Ihe foi atirado } \\
\text { (p.30f) - Sim / Não }\end{array}$ & \\
\hline & $\begin{array}{l}\text { Vítima de espancamento ou tentativa de estrangulamento (p.30g) } \\
\text { - Sim / Não }\end{array}$ & \\
\hline & Vítima de esfaqueamento ou tiro (p.30h) - Sim / Não & \\
\hline & Vítima de furto (p.23) - Sim / Não & \multirow{2}{*}{$\begin{array}{l}\text { VITPA } \\
\text { (Vitimização crimes } \\
\text { contra o patrimônio) }\end{array}$} \\
\hline & Vítima de roubo (p.24) - Sim / Não & \\
\hline \multirow{7}{*}{$\begin{array}{l}\text { Contato direto com } \\
\text { a polícia }\end{array}$} & Contato para pedido de informação (p.169a) - Sim / Não & \multirow{3}{*}{$\begin{array}{l}\text { CONTATO1 } \\
\text { (Contato direto: } \\
\text { assistência) }\end{array}$} \\
\hline & Contato para assistência/primeiros socorros (p.169b) - Sim / Não & \\
\hline & Contato para acidente de trânsito (p.169c) - Sim / Não & \\
\hline & Contato intervenção crime em andamento (p.169d) - Sim / Não & \multirow{2}{*}{$\begin{array}{l}\text { CONTATO2 } \\
\text { (Contato direto: } \\
\text { intervenção policial em } \\
\text { crime) }\end{array}$} \\
\hline & Contato comunicação de crime/violência (p.169e) - Sim / Não & \\
\hline & Contato para blitz (p.169f) & \multirow{2}{*}{$\begin{array}{l}\text { CONTATO3 } \\
\text { (Contato direto: atua- } \\
\text { ção ativa dos policiais) }\end{array}$} \\
\hline & Contato para revista pessoal (p.169g) & \\
\hline
\end{tabular}




\begin{tabular}{|c|c|c|}
\hline Dimensão & Variáveis integrantes & Fator gerado \\
\hline $\begin{array}{l}\text { Características } \\
\text { Sociodemográficas } \\
\text { da População }\end{array}$ & $\begin{array}{l}\text { Raça/cor } \\
\text { Escolaridade } \\
\text { Renda }\end{array}$ & $\begin{array}{c}\text { DEMO } \\
\text { (Características } \\
\text { Sociodemo- } \\
\text { gráficas) }\end{array}$ \\
\hline \multirow{7}{*}{$\begin{array}{l}\text { Percepção da atuação } \\
\text { ou contato indireto } \\
\text { com a polícia }\end{array}$} & $\begin{array}{l}\text { Presenciou PM apreendendo armas, drogas ou contrabando } \\
\text { (p.174a) - Sim / Não / Não sabe/Não lembra / Não respondeu }\end{array}$ & \\
\hline & $\begin{array}{l}\text { Presenciou enfrentamento armado entre polícia e tráfico, } \\
\text { gangues, fações ou milícias (p.174b) - Sim / Não / Não sabe/Não } \\
\text { lembra / Não respondeu }\end{array}$ & \multirow{6}{*}{$\begin{array}{l}\text { PPV } \\
\text { (Percepção da } \\
\text { atuação ou } \\
\text { contato indireto } \\
\text { com a polícia) }\end{array}$} \\
\hline & $\begin{array}{l}\text { Presenciou PM revistando veículos (p.174c) - Sim / Não / Não } \\
\text { sabe/Não lembra / Não respondeu }\end{array}$ & \\
\hline & $\begin{array}{l}\text { Presenciou PM fazendo revistas pessoais (p.174d) - Sim / Não / } \\
\text { Não sabe/Não lembra / Não respondeu }\end{array}$ & \\
\hline & $\begin{array}{l}\text { Presenciou perseguição policial (p.174e) - Sim / Não / Não sabe/ } \\
\text { Não lembra / Não respondeu }\end{array}$ & \\
\hline & $\begin{array}{l}\text { Presenciou atuação da PM em crimes que estavam acontecendo } \\
\text { (p.174f) - Sim / Não / Não sabe/Não lembra / Não respondeu }\end{array}$ & \\
\hline & $\begin{array}{l}\text { Presenciou pessoas sendo presas (p.174g) - Sim / Não / Não } \\
\text { sabe/Não lembra / Não respondeu }\end{array}$ & \\
\hline
\end{tabular}

Fonte: Questionário da Pesquisa Nacional de Vitimização - Senasp/MJ.

A própria dimensão da avaliação da qualidade da atuação das Polícias Militares foi transformada em um fator, composto a partir das variáveis descritas na Tabela 2.

A partir da formulação destes constructos, criou-se um modelo estatístico de regressão para mensurar os efeitos que cada um deles exercia sobre a avaliação da qualidade de atuação das Polícias Militares no Brasil. Para tanto, utilizou-se a técnica da Modelagem de Equações Estruturais (Structural Equation Modeling - SEM), procedimento que permite mensurar as múltiplas relações de dependência entre variáveis. Entretanto, a SEM apresenta particularidades em relação a modelos de mensuração simples ao incorporar a possibilidade de inclusão de variáveis latentes (cujos efeitos não podem ser mensurados diretamente) ao modelo. Neste sentido, a SEM é basicamente uma combinação de análises fatoriais com regressões múltiplas (Hair, 2009; Kelloway, 1998; Mackenzie, 2001; Jöreskog \& Sörbom, 1989)³.

Para a construção deste modelo estatístico, utilizou-se o software "AMOs", programa integrante do pacote IBM/SPSS. Em seus modelos de representação gráfica, o AMOS adota as seguintes convenções:

1. as variáveis observadas (isto é, as variáveis que reproduzem os valores obtidos mediante respostas dos entrevistados) são representadas por retângulos;

3. Ao longo dos últimos anos, a SEM se tornou umas das técnicas estatísticas mais usadas nas ciências sociais devido ao fato de possibilitar a mensuração de categorias teóricas complexas (através de construtos de variáveis diretas), possibilitando, inclusive, a predição de suas dimensões e valores. Além disso, a técnica também oferece a possibilidade de controlar os erros de mensuração, conferindo os níveis de confiança e de validade aos modelos (Mackenzie, 2001). 
TABELA 2

VARIÁVEIS COMPONENTES DO FATOR

"AVAliaÇÃo da Qualidade de ATUAÇÃo das Polícias Militares"

\begin{tabular}{|c|c|c|}
\hline Dimensão & Variáveis integrantes & Fator gerado \\
\hline \multirow{7}{*}{$\begin{array}{l}\text { Avaliação da qualidade } \\
\text { de atuação das Polícias } \\
\text { Militares }\end{array}$} & $\begin{array}{l}\text { PMs sabem como agir em situações de risco e perigo (p.171a)- } \\
\text { Concorda / Discorda / Nem concorda, nem discorda / Não sabe/ } \\
\text { Não lembra }\end{array}$ & \multirow{7}{*}{$\begin{array}{l}\text { AVA } \\
\text { (Avaliação da qualidade } \\
\text { de atuação das Polícias } \\
\text { Militares) }\end{array}$} \\
\hline & $\begin{array}{l}\text { PMs atendem as pessoas com cortesia, rapidez e segurança } \\
\text { (p.171b) - Concorda / Discorda / Nem concorda, nem discorda / } \\
\text { Não sabe/Não lembra }\end{array}$ & \\
\hline & $\begin{array}{l}\text { PMs abordam suspeitos de forma segura e dentro da lei (p.171c) } \\
\text { - Concorda / Discorda / Nem concorda, nem discorda / Não } \\
\text { sabe/Não lembra }\end{array}$ & \\
\hline & $\begin{array}{l}\text { PMs não estão preparados para usar armas de fogo (p.171d) - } \\
\text { Concorda / Discorda / Nem concorda, nem discorda / Não sabe/ } \\
\text { Não lembra }\end{array}$ & \\
\hline & $\begin{array}{l}\text { PMs abusam do uso da força e de sua autoridade (p.171e) - } \\
\text { Concorda / Discorda / Nem concorda, nem discorda / Não sabe/ } \\
\text { Não lembra }\end{array}$ & \\
\hline & $\begin{array}{l}\text { PMs fazem "vista grossa" à desonestidade de seus colegas } \\
\text { (p.171f) - Concorda / Discorda / Nem concorda, nem discorda / } \\
\text { Não sabe/Não lembra }\end{array}$ & \\
\hline & $\begin{array}{l}\text { PMs são preconceituosos quando abordam as pessoas na rua } \\
\text { (p.171g) - Concorda / Discorda / Nem concorda, nem discorda / } \\
\text { Não sabe/Não lembra }\end{array}$ & \\
\hline
\end{tabular}

Fonte: Questionário da Pesquisa Nacional de Vitimização - Senasp/MJ.

4. Ainda são representados no diagrama os erros de estimação das regressões e os erros específicos de cada variável na criação do fator, respectivamente esses erros estão representados pelas letras " $r$ " e " $e$ ".
2. as variáveis latentes (impossíveis de observação direta e, portanto, criadas através constructos advindos de variáveis observadas) são simbolizadas por elipses (assim como os erros);

3. setas em curva, com duas pontas, representam a covariância existente entre as variáveis;

4. setas retas representam as relações causais entre as variáveis (Maruyama, 1998) ${ }^{4}$.

A partir destas convenções, o modelo proposto neste artigo assumiu a seguinte representação gráfica: 
FIGURA 2

Modelo PARA MENSURAÇÃO DE EFEITOS DE VARIÁVEIS SOBRE

"AVALIAÇÃO DA QUALIDADE DA ATUAÇÃO

DAS POLÍCIAS MILITARES NO BRASIL"

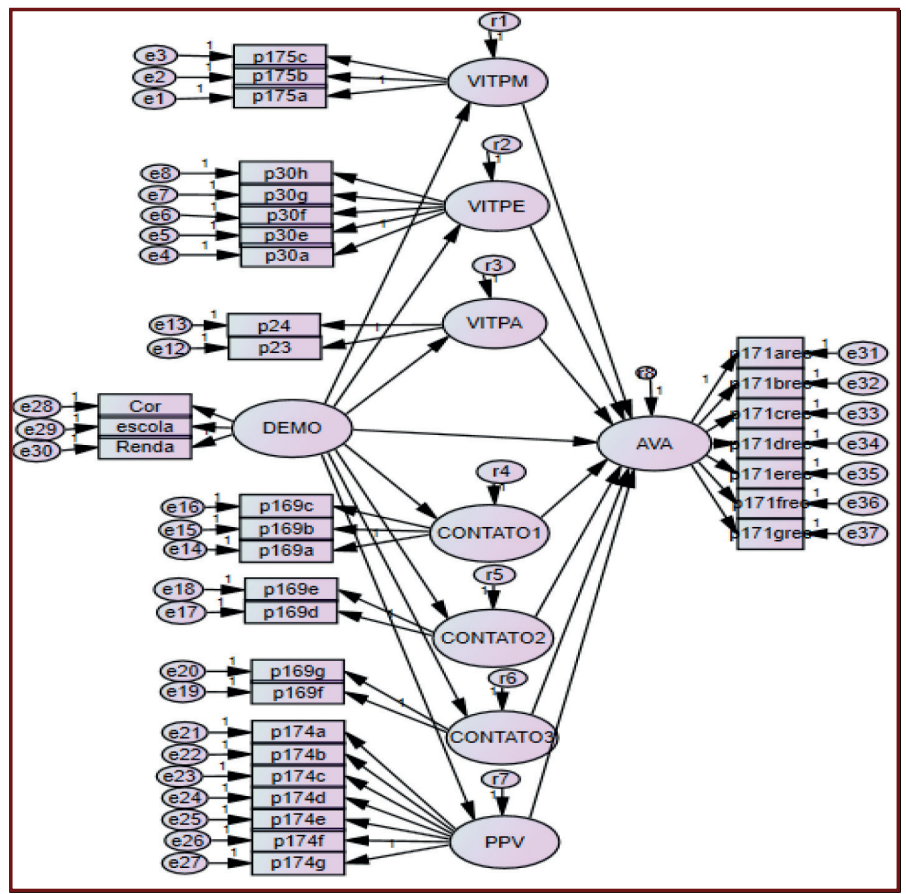

\section{Análise dos resultados}

Conforme mencionado anteriormente, o modelo aqui construído busca entender os efeitos que as variáveis explicativas (DEMO, VITPM, VITPE, VITPA, CONTATO1, CONTATO2, CONTATO3 E PPV) exercem sobre a avaliação da qualidade do trabalho das Polícias Militares no Brasil (AVA). Trata-se, portanto, de compreender o quanto a avaliação que a população faz do trabalho das PMs pode ser explicada por suas características sociodemográficas, ou pelo fato de terem sido vítimas de crimes ou da própria PM, ou ainda pelo contato direto ou indireto que mantiveram com policiais militares. Uma das possibilidades da análise SEM é mensurar, simultaneamente, os efeitos diretos e indiretos das variáveis presentes no modelo. Neste sentido, torna-se possível calcular não apenas o efeito direto da variável DEMO em AVA (DEMO $\rightarrow$ AVA), por exemplo, mas também os efeitos indiretos, diante da existência de uma variável interveniente (DEMO $\rightarrow \mathrm{VITPE} \rightarrow \mathrm{AVA}$ ). 
Ao tomar por base o modelo causal (apresentado no quadro a seguir) observa-se as decomposições dos efeitos diretos, indiretos e total de todo o modelo. Tendo como referência a variável exógena AVA, apenas a variável DEMO possui efeitos diretos e indiretos no modelo proposto. Nas outras interações, dado a inexistência de efeitos indiretos, os efeitos diretos são iguais aos efeitos totais. Deste modo, as equações das regressões no modelo proposto apresentaram-se da seguinte forma:

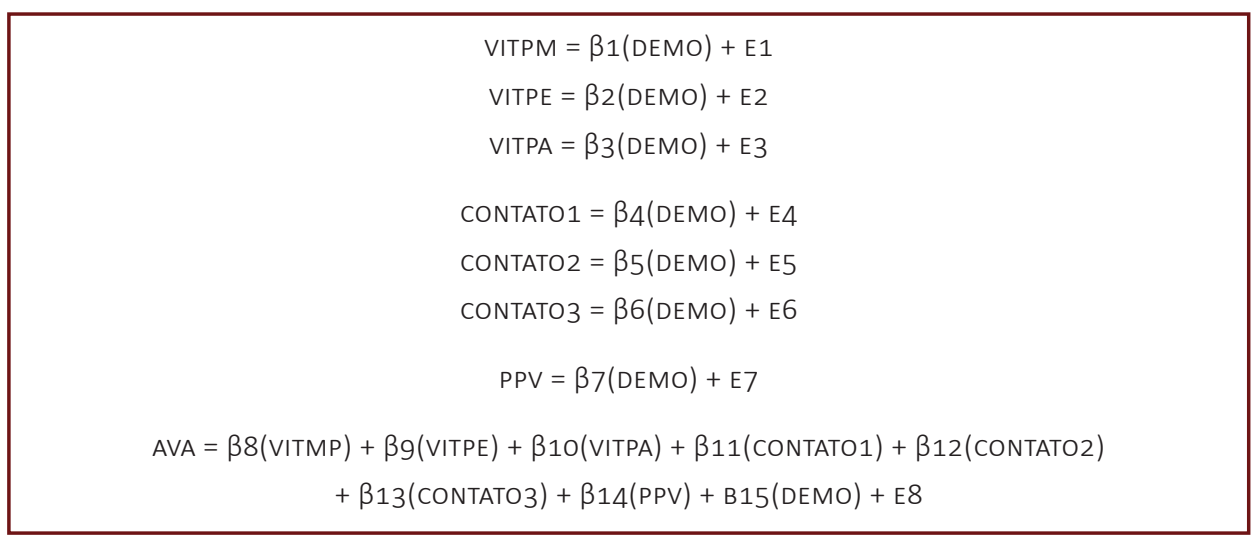

Para facilitar o entendimento da análise, optou-se aqui por apresentar o modelo causal e o modelo de mensuração em duas tabelas diferentes. No entanto, é importante lembrar que os modelos construídos por meio da SEM levam em consideração os dois tipos de modelos em um único cálculo. Tendo essa divisão em mente, cabe apenas observar que a Tabela 3 mostra que os parâmetros estimados pela análise fatorial são todos estatisticamente diferentes de zero em um nível de significância 0,001 (bicaudal). Já a Tabela 4 mostra a decomposição dos efeitos diretos, indiretos e total do modelo.

Antes de analisar os resultados do modelo propriamente dito, cabe fazer uma observação sobre a lógica de construção dos fatores cujos efeitos se pretendeu mensurar: os fatores de vitimização (VITPE, VITPA E VITPM), contato (CONTATO1, CONTATO2 e CONTATO3) e de percepção da ação policial na vizinhança (PPV) foram construídos seguindo a lógica de que quanto maior o escore, menor é a incidência do evento. Ou seja, quanto maior for o valor do escore obtido pelo fator VITPM, menor terá sido o grau de vitimização sofrido pela população por parte da Polícia Militar. A mesma lógica foi aplicada para o constructo DEMO (formado a partir de características sociodemográficas da população): quanto maior o valor do escore, menores os níveis de renda da população, menor seu grau de escolaridade formal e maior a proporção de "não brancos" (pretos ou pardos) entre os amostrados. 
TABELA 3

PARÂMETROS ESTIMADOS

PARA O MODELO DE MENSURAÇÃO

PROPOSTO NO MODELO

DE EQUAÇÕES ESTRUTURAIS

\begin{tabular}{|c|c|c|c|}
\hline \multicolumn{3}{|c|}{$\begin{array}{l}\text { Modelo de mensuração, } \\
\text { coeficiente }^{1}\end{array}$} & Parâmetros ${ }^{2}$ \\
\hline DEMO & $\rightarrow$ & RENDA & $0,641^{* * *}$ \\
\hline DEMO & $\rightarrow$ & ESCOLA & $0,670 * * *$ \\
\hline DEMO & $\rightarrow$ & COR & $0,200 * * *$ \\
\hline VITPM & $\rightarrow$ & p175a & $0,675^{* * *}$ \\
\hline VITPM & $\rightarrow$ & p175b & $0,858^{* * *}$ \\
\hline VITPM & $\rightarrow$ & p175c & $0,337 * * *$ \\
\hline VITPE & $\rightarrow$ & p30a & $0,395^{* * *}$ \\
\hline VITPE & $\rightarrow$ & p30e & $0,654^{* * *}$ \\
\hline VITPE & $\rightarrow$ & p30f & $0,458 * * *$ \\
\hline VITPE & $\rightarrow$ & p30g & $0,521 * * *$ \\
\hline VITPE & $\rightarrow$ & p30h & $0,200 * * *$ \\
\hline VITPA & $\rightarrow$ & p23 & $0,408 * * *$ \\
\hline VITPA & $\rightarrow$ & p24 & $0,392 * * *$ \\
\hline CONTATO1 & $\rightarrow$ & p169a & $0,498 * * *$ \\
\hline CONTATO1 & $\rightarrow$ & $\mathrm{p} 169 \mathrm{~b}$ & $0,528 * * *$ \\
\hline CONTATO1 & $\rightarrow$ & p169c & $0,516 * * *$ \\
\hline CONTATO2 & $\rightarrow$ & p169d & $0,470 * * *$ \\
\hline CONTATO 2 & $\rightarrow$ & p169e & $0,563^{* * *}$ \\
\hline CONTATO3 & $\rightarrow$ & $\mathrm{p} 169 f$ & $0,971 * * *$ \\
\hline CONTATO3 & $\rightarrow$ & $\mathrm{p} 169 \mathrm{~g}$ & $0,369 * * *$ \\
\hline PPV & $\rightarrow$ & p174a & $0,609 * * *$ \\
\hline PPV & $\rightarrow$ & p174b & $0,517 * * *$ \\
\hline PPV & $\rightarrow$ & $\mathrm{p} 174 \mathrm{c}$ & $0,642 * * *$ \\
\hline PPV & $\rightarrow$ & p174d & $0,697 * * *$ \\
\hline PPV & $\rightarrow$ & $\mathrm{p} 174 \mathrm{e}$ & $0,641 * * *$ \\
\hline PPV & $\rightarrow$ & $p 174 f$ & $0,606 * * *$ \\
\hline PPV & $\rightarrow$ & $\mathrm{p} 174 \mathrm{~g}$ & $0,716^{* * *}$ \\
\hline AVA & $\rightarrow$ & p171arec & $0,579 * * *$ \\
\hline AVA & $\rightarrow$ & p171brec & $0,669 * * *$ \\
\hline AVA & $\rightarrow$ & p171crec & $0,676 * * *$ \\
\hline AVA & $\rightarrow$ & p171drec & $0,503 * * *$ \\
\hline AVA & $\rightarrow$ & p171erec & $0,711 * * *$ \\
\hline AVA & $\rightarrow$ & p171frec & $0,657 * * *$ \\
\hline AVA & $\rightarrow$ & p171grec & $0,686 * * *$ \\
\hline
\end{tabular}

Fonte: Pesquisa nacional de vitimização, 2010-2012. 1 - O nível

de significância demostrados acima são soluções padronizadas

$\left({ }^{*} p, 0.05,{ }^{* *} p, 0.01,{ }^{* * *} p, 0.001\right) .2$ - 0 ajuste do modelo possui

os mesmos valores expostos na Tabela 4 .
TABELA 4

PARÂMETROS ESTIMADOS

PARA O MODELO CAUSAL

PROPOSTO NO MODELO DE

EQUAÇÕES ESTRUTURAIS

\section{Modelo causal, coeficiente Parâmetros padronizado, $(95 \% \text { ic })^{1} \quad$ estimados $^{2}$}

Efeito total

\begin{tabular}{|c|c|c|c|}
\hline DEMO & $\rightarrow$ & AVA & $-0,146 * * *$ \\
\hline \multicolumn{4}{|c|}{ Efeito Direto } \\
\hline DEMO & $\rightarrow$ & VITPM & $-0,129 * * *$ \\
\hline DEMO & $\rightarrow$ & VITPE & $-0,061^{* * *}$ \\
\hline DEMO & $\rightarrow$ & VITPA & $-0,348 * * *$ \\
\hline DEMO & $\rightarrow$ & CONTATO1 & $-0,411 * * *$ \\
\hline DEMO & $\rightarrow$ & CONTATO2 & $-0,331 * * *$ \\
\hline DEMO & $\rightarrow$ & CONTATO3 & $-0,349 * * *$ \\
\hline DEMO & $\rightarrow$ & PPV & $-0,156 * * *$ \\
\hline DEMO & $\rightarrow$ & AVA & $-0,125 * * *$ \\
\hline VITPM & $\rightarrow$ & AVA & $0,201 * * *$ \\
\hline VITPE & $\rightarrow$ & AVA & $0,034 * * *$ \\
\hline VITPA & $\rightarrow$ & AVA & $0,111^{* * *}$ \\
\hline CONTATO1 & $\rightarrow$ & AVA & $-0,091^{* * *}$ \\
\hline CONTATO2 & $\rightarrow$ & AVA & $-0,038 * * *$ \\
\hline CONTATO3 & $\rightarrow$ & AVA & $-0,038 * * *$ \\
\hline PPV & $\rightarrow$ & AVA & $0,109 * * *$ \\
\hline \multicolumn{4}{|c|}{ Efeito indireto } \\
\hline DEMO & $\rightarrow$ & AVA & $-0,021 * * *$ \\
\hline \multicolumn{4}{|c|}{ Ajuste do Modelo } \\
\hline AGFI & & & 0,913 \\
\hline RMSEA & & & 0,046 \\
\hline
\end{tabular}

Fonte: Pesquisa nacional de vitimização, 2010-2012. O nivel de significância demostrados acima são soluções padronizadas $\left({ }^{*} p, 0.05\right.$, **p, $0.01, * * * p, 0.001)$ 
Dito isso, um primeiro aspecto a ser destacado no modelo são os três constructos de variáveis que apresentaram os maiores efeitos sobre a avaliação que a população faz do trabalho das Polícias Militares no Brasil. Por ordem do valor do efeito:

1. vitimização por parte das PMs;

2. configurações sociodemográficas;

3. vitimização para crimes contra o patrimônio.

Conforme demonstrado pelo modelo, a configuração sociodemográfica (DEMO) possui um efeito total bastante expressivo sobre a avaliação que a população faz do trabalho das PMs no Brasil $(-0,146)$. Sendo que a quase totalidade desse efeito é direto $(-0,125)$ e não intermediado pelas demais variáveis presentes no modelo $(-0,021)$, como poderia se pensar inicialmente. De modo geral, o que os dados indicam é que segmentos populacionais com menores níveis de renda, com menor escolaridade e de raça/cor "não branca" (pretos e/ou pardos) tendem a avaliar pior o trabalho executado pelas polícias militares (o aumento de um desvio padrão na variável DEMO provoca uma queda de 0,146 desvio padrão na variável AVA).

Outro aspecto importante destacado pelo modelo são os efeitos que a vitimização (criminal e/ou institucional) projeta sobre a avaliação do trabalho das PMs. Dentre todas as variáveis mensuradas, a violência e os desvios praticados pelos próprios policiais (agressões físicas e/ou verbais e extorsões/propinas) são as que possuem maior poder preditivo sobre a avaliação que a população faz do trabalho das Polícias Militares no Brasil. Para cada desvio padrão aumentado em VITPM aumenta-se 0,201 desvio padrão de AVA. Ou seja, quando se diminui a incidência de desvios policiais, aumenta-se consideravelmente a avaliação positiva que a população faz do trabalho das PMs.

A vitimização provocada pelos próprios policiais militares não é apenas o fator de maior relevância entre todos os tipos de vitimização, como também é o constructo com maior efeito entre todas as variáveis mensuradas no modelo aqui proposto. Quando comparado com o efeito das demais variáveis, o efeito de VITPM é pelo menos duas vezes maior do que o efeito de qualquer variável de contato direto (CONTATO1, CONTATO2 e CONTATO3), ou mesmo da variável de contato indireto, que trata da percepção da atuação de policiais militares na vizinhança (PPV). O efeito de vitimizações praticadas pela PM chega a ser pelo menos cinco vezes maior que o efeito da vitimização para crimes contra a pessoa (VITPE) e quase duas vezes maior que a vitimização para crimes contra o patrimônio (VITPA). 
Para os demais constructos de vitimização (crimes contra a pessoa e contra o patrimônio), manteve-se a lógica de que quanto menos vitimada é a pessoa, melhor será a avaliação que ela fará do trabalho das Polícias Militares. No entanto, cabe chamar a atenção para a disparidade de resultados obtida na comparação entre crimes contra a pessoa (VITPE) e crimes contra o patrimônio (VITPA). Enquanto VITPE possui um efeito direto de 0,034 sobre as avaliações, VITPA apresentou um efeito de 0,111 (um efeito três vezes maior). Ou seja, o modelo indica que, de modo geral, a população tende a avaliar a qualidade do trabalho das PMs muito mais em função da sua capacidade de prevenir, ou de dar solução, para crimes contra o patrimônio do que crimes contra a pessoa. Possivelmente porque, em muitos casos de crimes contra a pessoa (constructo composto por variáveis de agressões verbais e físicas), a vítima assuma papel ativo no conflito que gerou a vitimização, ao contrário do que ocorre nos crimes contra o patrimônio.

No que diz respeito especificamente aos fatores que tratam do contato da população com Policiais Militares (CONTATO1/Assistência; cONTATO2/Intervenção em crime; CONTATO3/Atuação ativa dos policiais), observa-se que, embora estatisticamente significantes, nenhuma das modalidades de contato possui grande efeito sobre a avaliação da ação policial. Ainda assim, constata-se que os três fatores projetam o mesmo tipo de efeito: quanto maior o contato dos entrevistados com policiais, maior é o escore positivo da avaliação (AVA $)^{5}$.

Em princípio, o padrão identificado a partir dos dados da PNV (de quanto maior o contato entre cidadãos e policiais, melhor a avaliação popular sobre a qualidade do trabalho das PMs), poderia levar à interpretação de que o resultado aqui obtido vai na direção oposta às tendências observadas em estudos internacionais. No entanto, cabe observar que, ao contrário do que geralmente ocorre em outras pesquisas do gênero, o questionário da PNV foi construído de modo a permitir a especificação das modalidades de contato mantidos entre cidadãos e policiais. Ao invés de trabalhar com uma variável geral de "contato com a polícia", as questões permitiram estabelecer constructos sobre tipos específicos de contato e mensurar individualmente seus efeitos sobre a avaliação que a população faz do trabalho policial.

É nesse sentido que foi possível, por exemplo, separar os contatos típicos de "vitimização por parte da polícia" (que projetam forte impacto sobre a avaliação do trabalho policial) das demais modalidades de interação entre cidadãos e policiais. Não por acaso, as modalidades de contato nas quais os agentes policiais prestaram socorro às vítimas de acidentes de trânsito, ou prestaram informações quando solicitados, tiveram maior impacto positivo sobre a avaliação popular do que os contatos mantidos por meio da realização de blitz.
5. Cabe ressaltar que os contatos entre cidadãos e policiais referentes à prestação de serviços de primeiros socorros, informação ou atendimento a acidente de trânsito (CONTATO1) possuem, em conjunto, um efeito quase três vezes maior na avaliação da ação policial do que os outros dois tipos de contato. 
Por fim, o modelo também demonstra que a percepção da ação policial na vizinhança (PPV - contato indireto) provoca um efeito negativo na avaliação que as pessoas fazem da qualidade do trabalho das PMs. Ou seja, quanto maior a presença de ações policiais nas comunidades nas quais o entrevistado reside, pior é a avaliação que ele faz do trabalho policial (o aumento de um desvio padrão da variável PPV, isto é, o aumento da ausência da ação policial, aumenta 0,109 desvio padrão da variável AVA).

\section{Considerações finais}

Conforme mencionado anteriormente, o objetivo deste trabalho foi tentar compreender melhor como e com qual intensidade determinados elementos estruturais, criminais e perceptivos impactam a avaliação que a população brasileira faz sobre a qualidade do trabalho das Polícias Militares no Brasil. A partir de dados produzidos pela Pesquisa Nacional de Vitimização, buscou-se mensurar em qual grau a avaliação popular sobre o trabalho das PMs é impactada por diferentes configurações sociodemográficas, variados níveis de vitimização criminal e/ou institucional, bem como por diversos modos de contato com a polícia e de percepção da própria atuação policial nas vizinhanças.

Em sentido mais amplo, a presente investigação dialoga com uma vasta produção acadêmica que, ao longo dos últimos anos, vem tentando escrutinar aspectos diversos da relação entre sociedade civil e instituições de segurança pública no Brasil. No caso específico das organizações policiais, identificar os fatores que, em maior ou menor grau, determinam a avaliação que a população faz sobre a qualidade de seus trabalhos constitui condição importante para compreender também os níveis de confiança e de legitimidade socialmente atribuídos a elas. Em última instância, pode ajudar a explicar os níveis de efetividade das corporações policiais no controle do crime e da violência, na medida em que são justamente tais percepções as principais responsáveis por conformar padrões atitudinais de maior ou menor colaboração entre a população e as forças de segurança pública.

De modo geral, os resultados obtidos pela presente pesquisa apontam basicamente na mesma direção de grande parte dos estudos já produzidos sobre o tema no exterior: a de que a avaliação que a população brasileira faz da qualidade do trabalho das Polícias Militares orienta-se muito mais por questões relacionadas ao controle da própria atividade policial e aos modos de relacionamento das corporações com suas comunidades e com segmentos populacionais específicos do que propriamente por sua efetividade na redução dos índices de vitimização criminal. 
De longe, as variáveis com maior efeito sobre a avaliação que as pessoas fazem sobre a qualidade do trabalho das PMs no Brasil foram as de vitimização policial (quando policiais praticam agressões físicas, verbais e extorsão/propina), seguidas por características sociodemográficas associadas a públicos tradicionalmente vulneráveis ("não brancos", com baixa renda e escolaridade). Tais achados corroboram, em larga medida, estudos realizados ao longo dos últimos anos nos Estados Unidos e na Europa, segundo os quais a percepção pública sobre a qualidade do trabalho das corporações (positiva ou negativa) tem como seus principais determinantes o contato direto da população com agentes policiais, bem como as características sociodemográficas das comunidades atendidas (Brown \& Benedict, 2002; Frank et alii, 2005; Larsen \& Blair, 2009; Avdija, 2010).

Já em termos criminais, a redução da vitimização para crimes contra o patrimônio mostrou ter um impacto muito mais forte sobre a avaliação do que a vitimização para crimes contra a pessoa. Ainda assim, ambas as categorias apresentaram efeitos reduzidos sobre a variável de avaliação, principalmente quando comparados aos impactos provocados pela vitimização direta por parte da polícia. Estes resultados também se alinham a conclusões obtidas por outros estudos, inclusive alguns realizados no Brasil, segundo os quais experiências de vitimização criminal ajudam a moldar a forma como a população avalia a qualidade do trabalho das forças policiais. No entanto, são as variáveis de contato direto com os agentes policiais (seja qual for sua natureza) que possuem maior poder de determinação sobre tais percepções (Lopes, 2010; Silva \& Beato, 2013).

O que a análise aqui desenvolvida indica, portanto, é que parte significativa da meIhoria da avaliação popular com relação ao trabalho das PMs no Brasil (e, consequentemente, o próprio aumento da legitimidade e a melhoria do serviço prestado pelas organizações) passa necessariamente pelo enfrentamento mais rigoroso e dedicado de questões localizadas unicamente na esfera institucional das próprias Polícias Militares. Entre elas, destacam-se o incremento do controle da atividade policial (combate à violência praticada pelos próprios PMs) e o desenvolvimento de melhores estratégias de aproximação e de relacionamento com segmentos populacionais específicos, historicamente mais vitimizados pela violência institucional nos grandes centros urbanos e, como os dados indicam, pelas próprias organizações. 
To serve and protect:

determinants of public evaluation about

the quality of Military Police work in Brazil

Abstract: Inserted in the field of the relationship between civil society and public security institutions, this article aims to measure and analyze the influence that certain types of variables (eg, sociodemographic features, criminal or institutional victimization experiences, contact with institutions, etc.) have on the assessment that the Brazilian population make about the quality of Military Police (MP) work. To do so, we use data produced by the National Victimization Survey (NVS), sample study conducted between 2010 and 2012 in all Brazilian states. In general, NVS's data indicate that, contrary to common sense, the fact of being a crime victim exerts relatively little influence on the perception that people have about the quality of Military Police work. The variables that had the greatest effect on the evaluation (positive or negative) about MP's work were those related to having been victim of violence or extortion practiced by the military itself, as well as the experiences of direct contact with the police. These findings reinforce the hypothesis (already well known in international literature) that positive or negative evaluations about police work are not exclusively and necessarily linked to the perception that such corporations are more or less effective in preventing crime, but to issues located only in institutional sphere of these organizations, such as the fight against violence and corruption perpetrated by the MPs and the improvement of its relationship with most vulnerable segments of the population.

Keywords: organizations, evaluation and accountability, Military Police, public policy.

\section{Referências}

AVDIJA, Avdi S. The role of police behavior in predicting citizen's attitudes toward the police. Applied Psychology in Criminal Justice, v. 2, n. 6, p. 76-90, 2010.

BAYLEY, D. H.; SKOLNICK, J. Nova polícia: inovações na polícia de seis cidades norte-americanas. São Paulo: EdUSP, 2001.

BELLI, B. Violência policial e segurança pública: democracia e continuidade autoritária no Brasil contemporâneo. Impulso, v. 15, n. 37, p. 17-34, 2004.

BROWN, B.; BENEDICT, W. R. Perceptions of the police: past findings, methodological issues, conceptual issues and policy implications. Policing: an International Journal of Police Strategies and Management, v. 25, n. 3, p. 543-580, 2002.

CANO, I. Controle de polícia no Brasil. São Paulo: Instituto Sou da Paz, 2006. Disponível em: <http://www.altus.org/pdf/b_ic_pt.pdf>. Acessado em 28 Maio 2014.

COSTA, Arthur Trindade; LIMA, Renato Sérgio. Segurança pública. In: LIMA, Renato Sérgio; RATTON, José Luiz; GHIRINGHELLI, Rodrigo (Orgs.). Crime, polícia e justiça no Brasil. São Paulo: Contexto, 2014. 
FRANK, J.; SMITH, B.; NOVAK, K. Exploring the basis of citizens' attitudes toward the police. Police Quartely, v. 8, n. 2, p. 206-228, Jun. 2005.

GOLDSMITH, Andrew. Police reform and the problem of trust. Theoretical Criminology, v. 9, n. 4, p. 443-470, 2005.

GONÇALVES, Alcindo. O conceito de governança. Trabalho apresentado no XIV Congresso Nacional do Conselho Nacional de Pesquisa e Pós-Graduação em Direito (Conpedi), Fortaleza, 2005

HAIR, Joseph F. et alii. Análise multivariada de dados. Porto Alegre: Bookman, 2009.

IVKOVIC, S. K. A. A comparative study of public support for the police. International Criminal Justice Review, v. 18, n. 4, p. 406-434, Dec. 2008.

JÖRESKOG, K. G.; SÖRBOM, D. LISREL: A guide to the program and applications. Sweden: Uppsala, 1989.

KELLOWAY, E. K. Using LISREL for structural equation modeling. Thousand Oaks (CA): Sage Publications, 1998.

LARSEN, J. E.; BLAIR, J. P. The importance of police performance as a determinant of satisfaction with the police. American Journal of Economics and Business Administration, v. 1, n. 1, p. 1-10, 2009.

LOPES, C. S. Por que os brasileiros desconfiam da polícia? Uma análise das causas da desconfiança na instituição policial. Texto apresentado no VII Encontro da ABCP, Recife, 2010.

MACKENZIE, S. B. Opportunities for improving consumer research through latent variable structural equation modeling. Journal of Marketing Research, v. 28, n. 1, p. 159-166, 2001.

MARUYAMA, G. M. Basics of structural equation modeling. London: Sage Publications, 1998.

MINISTÉRIO DA JUSTIÇA; CRISP/UFMG; DATAFOLHA (2013) Relatório final da Pesquisa Nacional de Vitimização. Disponível em <http://www.crisp.ufmg.br/wp-content/ uploads/2013/10/Relatório-PNV-Senasp_final.pdf>. Acessado em 21 Set. 2015.

MOISÉS, J. A. A desconfiança nas instituições democráticas. Opinião Pública, v. 11, n. 1, p. 33-63, 2005. 
OLIVEIRA JÚNIOR, Almir. Dá pra confiar nas polícias? Confiança e percepção social da polícia no Brasil. Revista Brasileira de Segurança Pública. Ano 5, ed. 9, p. 6-22, Ago./Set. 2011.

SHERMAN, Lawrence W.; GOTTFREDSON, Denise C.; MCKENZIE, Doris L.; ECK, John; REUTER, Peter; BUSHWAY, Shawn D. Preventing crime: what works, what doesn't, and what promising. Washington: National Institute of Justice (NIJ), 1998.

SILVA, Geélison; BEATO, Cláudio. Confiança na polícia em Minas Gerais: o efeito da percepção de eficiência e do contato individual. Opinião Pública, v. 19, n. 1, p. 118153, Jun. 2013.

SOARES, Luiz Eduardo. Meu casaco de general: quinhentos dias no front da segurança pública do Rio de Janeiro. São Paulo: Companhia das Letras, 2000.

SOUZA, Celina. Políticas públicas: uma revisão da literatura. Sociologias, Ano 8, n. 16, p. 20-45, Jul./Dez. 2006.

TYLER, T. R. Public trust and confidence in legal authorities: what do majority and minority group members want from the law and legal institutions? Behavioral Sciences and the Law, n. 19, p. 215-235, 2001.

VELÁSQUEZ, Hugo Acero. Os governos locais e a segurança cidadã. Washington: United Nations Development Program, 2006.

VELOSO, Fernando; FERREIRA, Sérgio Guimarães. Mecanismos de gestão em segurança Pública. Texto preparado para o Grupo Rio. Rio de Janeiro, 2007.

WEISBURD, David; BRAGA, Anthony. La diffusion de l'innovation dans la police. Quelles leçons retenir de l'expérience américaine? Revue Française de Science Politique, v. 59, n. 6, p. 1097-1126, Dez. 2009. 